\title{
A GLOBAL MORPHISM FROM THE DOUADY SPACE TO THE CYCLE SPACE
}

\author{
JÓN INGÓLFUR MAGNÚSSON
}

\begin{abstract}
We establish, for any given complex space $M$, a global morphism from the reduction of its Douady space to its cycle space. This morphism is an extension of the morphism defined in [1] from the subspace of the Douady space formed by all pure dimensional subspaces of $M$ to the cycle space of $M$. In the case where $M$ is projective this morphism is the classical morphism from the Hilbert scheme of $M$ to the Chow scheme of $M$.
\end{abstract}

\section{Introduction}

Let $M$ be a complex space. Then there is a natural map from its Douady space to its cycle space that maps every compact complex subspace of $M$ to its fundamental cycle. The object of the present paper is to prove that this map is a holomorphic map from the reduction of the Douady space of $M$ to the cycle space of $M$. See corollary 3.3.

This result is known in the case where $M$ is projective since in that case there is a global morphism from the Hilbert scheme of $M$ to the Chow scheme of $M$ (see [6]), and in that case it is easy to see that the Douady space of $M$ is the complex space associated with the Hilbert scheme of $M$ and from [1] it is known that the cycle space of $M$ is the complex space associated with the Chow scheme of $M$.

For a general complex space $M$ a weaker version of this result can be found in [1], where it is proved that the restriction of the above map to the reduction of the subspace of the Douady space of $M$ consisting of all pure dimensional subspaces (having no embedded components) is holomorphic.

We will obtain this result as a simple consequence of the following more general theorem. See section 1 for notations and terminology.

MAIN Theorem. Let $M$ be a complex space and let $\mathscr{Z} \subset S \times M$ be a flat family of n-dimensional subspaces of $M$. Let $q: \mathscr{Z} \rightarrow M$ and $\pi: \mathscr{Z} \rightarrow S$ denote the canonical projections and for each $s$ in $S$ let $\mathscr{Z}_{s}$ denote the fibre

Received September 9, 2005. 
of $\pi$ over $s$ and put $Z_{s}:=q\left(\mathscr{Z}_{s}\right)$. Then $\left(\left[Z_{s}\right]\right)_{s \in S_{\text {red }}}$ is an analytic family of $n$-cycles in $M$.

In section 1 we introduce the basic definitions and preliminary results needed. Then a special case will be proved in section 2 and finally we prove the Main Theorem in section 3.

The proof is based on the same ideas as in [1].

\section{Basic notions and preliminaries}

In this section $M$ will denote a (not necessarily reduced) complex space.

\subsection{Basic definitions}

Definition 1.1. A flat family of subspaces of $M$ is a pair of complex spaces $(S, \mathscr{Z})$ such that $\mathscr{Z}$ is a subspace of $S \times M$ and such that the natural projection $\pi: \mathscr{Z} \rightarrow S$ is flat. If the projection is also a proper map then the family is called flat and proper.

Definition 1.2. An $n$-cycle in $M$ is a locally finite linear combination

$$
Z=\sum_{i \in I} n_{i} Z_{i}
$$

with coefficients in $\mathrm{N}^{*}$, where the $Z_{i}$ are globally irreducible complex subspaces of $M$ of dimension $n$ such that $Z_{i} \neq Z_{j}$ holds for $i \neq j$. The set

$$
|Z|:=\bigcup_{i \in I} Z_{i}
$$

is called the support of $Z$. The $n$-cycle $Z$ is called compact if its support $|Z|$ is compact.

Definition 1.3. A scale of $M$ is a triplet $E=(U, B, j)$ having the following properties:

(i) $U \Subset \mathrm{C}^{n}$ and $B \Subset \mathrm{C}^{p}$ are open polydisks,

(ii) $j$ is a holomorphic embedding of an open subset $M_{E}$ of $M$ into an open neighbourhood of $\bar{U} \times \bar{B}$ in $\mathrm{C}^{n+p}$.

The scale $E$ is said to be adapted to an $n$-cycle $Z$ if

$$
j\left(|Z| \cap M_{E}\right) \cap(\bar{U} \times \partial B)=\emptyset .
$$

The $k$-th symmetric group acts on $\left(\mathrm{C}^{p}\right)^{k}=\mathrm{C}^{p} \times \cdots \times \mathrm{C}^{p}$ by permutation

$$
\beta\left(x_{1}, \ldots, x_{k}\right):=\left(x_{\beta(1)}, \ldots, x_{\beta(k)}\right) .
$$


The orbit space of this action is called the $k$-th symmetric power of $\mathrm{C}^{p}$ and will be denoted by $\operatorname{Sym}^{k}\left(C^{p}\right)$. It is a normal complex space. (See for instance [5]).

The $k$-th symmetric group acts in the same way on $B^{k}$ and the orbit space $\operatorname{Sym}^{k}(B)$ can be naturally identified with an open subset of $\operatorname{Sym}^{k}\left(C^{p}\right)$.

Assume $E=(U, B, j)$ is a scale of a complex space $M$ adapted to an $n$-cycle $Z$ in $M$. Then $Z$ induces a ramified covering of a certain degree of an open neighborhood of $\bar{U}$ whose degree will be denoted by $\operatorname{deg}_{E} Z$ or $k_{E}$ for short. Hence $Z$ induces a holomorphic map

$$
U \rightarrow \operatorname{Sym}^{k_{E}}(B) .
$$

For a detailed discussion see [1] or [7].

DEFINITION 1.4. Let $S$ be a reduced complex space and let $\left(Z_{S}\right)_{s \in S}$ be a family of $n$-cycles in $M$.

(i) The family $\left(Z_{S}\right)_{s \in S}$ is called analytic if for every $s_{0} \in S$ and every scale $E=(U, B, j)$, adapted to the $n$-cycle $Z_{s_{0}}$, there exists an open neighbourhood $S_{E}$ of $s_{0}$ in $S$ such that

(a) $E$ is adapted to $Z_{s}$ for all $s \in S_{E}$,

(b) $\operatorname{deg}_{E} Z_{s}=\operatorname{deg}_{E} Z_{s_{0}}$ for all $s \in S_{E}$,

(c) the map $g_{E}: S_{E} \times U \rightarrow \operatorname{Sym}^{k_{E}}(B)$ is holomorphic, where $g_{E}(s, \cdot): U \rightarrow \operatorname{Sym}^{k_{E}}(B)$ is the holomorphic map induced by $Z_{s}$.

(ii) The family $\left(Z_{S}\right)_{s \in S}$ is called a proper analytic family of compact $n$-cycles if it is analytic, every cycle is compact and for every $s_{0} \in S$ and every neighborhood $W$ of $\left|Z_{s_{0}}\right|$ there exists an open neighborhood $S_{0}$ of $s_{0}$ in $S$ such that $\left|Z_{s}\right| \subset W$ for all $s \in S_{0}$.

REMARK 1.5 . For a family $\left(Z_{s}\right)_{s \in S}$ of $n$-cycles in $M$ the set

$$
G:=\left\{(s, z) \in S \times M|z \in| Z_{s} \mid\right\}
$$

is usually called the graph of the family and it is easy to see that the topological condition in (ii) is verified if and only if the canonical projection $G \rightarrow S$ is a proper mapping.

Let $Z$ be an irreducible component of $M$. Then $\stackrel{\circ}{Z}:=Z \backslash \operatorname{Sing}\left(M_{\text {red }}\right)$ is a connected manifold and we have $\mathscr{O}_{\dot{Z}, z}=\mathscr{O}_{M_{\text {red }}, z}$ for all $z \in \stackrel{Z}{Z}$. For each $z \in \stackrel{\circ}{Z}$ the analytic algebra $\mathscr{O}_{\tilde{Z}, z}$ is regular so there exists a section

$$
\mathcal{O}_{M, z} \longleftarrow \mathcal{O}_{\dot{Z}, z}
$$

which makes $\mathscr{O}_{M, z}$ a coherent $\mathscr{O}_{\grave{Z}, z}$-module. The rank of this module is independent of the choice of the section as can be seen in the following way. 
Put

$$
\mathcal{O}:=\mathcal{O}_{M_{\text {red }}, z}
$$

and let $\mathfrak{n}$ denote the nilradical of $\mathcal{O}$. Then it is easy to show that the length of the localized ring $\mathscr{O}_{\mathfrak{n}}$ is equal to the rank of $\mathscr{O}$ as a $\mathscr{O}_{\text {red }}$-module.

It follows that the rank is the same for all points $z$ in $\stackrel{\circ}{Z}$ and it is called the multiplicity of the component $Z$ in $M$.

Now suppose that the complex space $M$ is of dimension $n$ and let $M^{\prime}$ denote the union of of the irreducible components of $M$ whose dimension is strictly less than $n$. Let $\bar{M}$ denote the analytic closure of $M \backslash M^{\prime}$ in $M$, in other words $\bar{M}$ is the smallest complex subspace of $M$ that contains $M \backslash M^{\prime}$. Then $\bar{M}$ is a complex space of pure dimension $n$.

Let $\left(\bar{M}_{i}\right)_{i \in I}$ be the family of the irreducible components of $\bar{M}$, in other words the irreducible components of dimension $n$ of $M$, and put

$$
[M]:=\sum_{i \in I} n_{i} \bar{M}_{i}
$$

where $n_{i}$ is the multiplicity of $\bar{M}_{i}$ in $M$.

Definition 1.6. The $n$-cycle $[M]$ is called the fundamental cycle of $M$.

\subsection{Preliminary results}

For every positive integer $l$ let $S^{l}\left(\mathrm{C}^{p}\right)$ be the $l$-th symmetric product of the complex vector space $\mathrm{C}^{p}$ and let $\sigma_{l}:\left(\mathrm{C}^{p}\right)^{k} \rightarrow S^{l}\left(\mathrm{C}^{p}\right)$ be the $l$-th elementary symmetric polynomial. One can interpret this mapping in the following way. Think of the elements in $\mathrm{C}^{p}$ as $\mathrm{C}$-linear forms on the dual space $\left(\mathrm{C}^{p}\right)^{\star}$, in other words think of them as homogeneous poynomials of degree 1 in $p$ variables. Then $S^{l}\left(C^{p}\right)$ becomes the space of homogeneous polynomials of degree $l$ in $p$ variables and $\sigma_{l}\left(v_{1}, \ldots, v_{k}\right)$ is a symmetric polynomial of degree $l$ in $p$ variables for every $l \in\{1, \ldots k\}$ and for every $\left(v_{1}, \ldots, v_{k}\right) \in\left(\mathrm{C}^{p}\right)^{k}$.

Proposition 1.7. The mapping

$$
S: \operatorname{Sym}^{k}\left(\mathrm{C}^{p}\right) \rightarrow \bigoplus_{l=1}^{k} S^{l}\left(\mathrm{C}^{p}\right)
$$

induced by $\left(\sigma_{1}, \ldots, \sigma_{k}\right)$ is a proper holomorphic embedding.

Proof. See [1] or [7].

THeOREM 1.8 (Douady). Let $M$ be a complex space. Then there exists a complex space $\mathscr{D}=\mathscr{D}(M)$ and a subspace $\mathscr{X} \subset \mathscr{D} \times M$, called the universal (flat and proper) family, having the following properties: 
(i) The canonical projection $\pi: \mathscr{X} \rightarrow \mathscr{D}$ is a flat and proper map.

(ii) (Universal property) If $(S, \mathscr{Z})$ is a flat and proper family of subspaces of $M$, then there exists a unique holomorphic map $f: S \rightarrow \mathscr{D}$ such that

$$
\mathscr{Z}=S \times \mathscr{D} \mathscr{X} .
$$

Proof. See [3].

The set of all compact $n$-cycles in $X$ will be denoted by $\mathscr{C}_{n}(M)$ and the set of all compact cycles in $M$ will be denoted by $\mathscr{C}(M)$. Obviously $\mathscr{C}(M)$ is the disjoint union of the family $\left(\mathscr{C}_{j}(M)\right)_{j \geq 0}$.

THEOREM 1.9 (Barlet). The set $\mathscr{C}_{n}(M)$ carries a reduced complex structure such that the following conditions are fulfilled:

(i) The family $(X)_{X \in \mathscr{C}_{n}(M)}$ is a proper analytic family of compact $n$-cycles.

(ii) (Universal property) For every analytic family $\left(Z_{s}\right)_{s \in S}$ of compact $n$ cycles the map

$$
S \rightarrow \mathscr{C}_{n}(M), \quad s \mapsto Z_{s}
$$

is holomorphic.

Proof. See [1].

The complex spaces $\mathscr{D}(M)$ and $\mathscr{C}(M)$ are called the Douady space and the (Barlet) cycle space of $M$.

\section{The case of dimension zero}

THeOREM 2.1. Let $T$ be a reduced complex space and let $B$ be a relatively compact open polydisk in $\mathrm{C}^{p}$. Let $\mathscr{Z} \subset T \times B$ be a flat and proper family of 0 -dimensional subspaces of $B$. Then the associated family of 0 -cycles in $B$ is analytic.

Proof. For each $t$ in $T$ let $Y_{t}$ denote the image of the fiber $\mathscr{Z}_{t}$ by the canonical projection $q: \mathscr{Z} \rightarrow B$, so that

$$
\mathscr{Z}_{t}=\{t\} \times Y_{t} .
$$

Let $\mathscr{A}^{t}$ be the structure sheaf of the zero-dimensional subspace $Y_{t}$ of $B$ and let $X_{t}$ be the fundamental cycle of $Y_{t}$. Then $X_{t}$ is given by the formula

$$
X_{t}:=\sum_{y \in Y_{t}} n_{y}(t)\{y\}
$$

where $n_{y}(t):=\operatorname{dim}_{\mathrm{C}} \mathscr{A}_{y}^{t}$. 
The canonical projection $\pi: \mathscr{Z} \rightarrow T$ is flat and finite so $\pi_{*} \mathscr{O}_{\mathscr{Z}}$ is a locally free $\mathscr{O}_{T}$-module. Without loss of generality we may assume $T$ connected and consequently $\pi_{*} \mathcal{O}_{\mathscr{Z}}$ of constant rank which will be denoted by $k$. For every $t$ in $T$ we then have

$$
\left(\pi_{*} \mathcal{O}_{\mathscr{Z}}\right)_{t}=\bigoplus_{y \in Y_{t}} \mathscr{O}_{\mathscr{Z},(t, y)}
$$

and

$$
\mathscr{A}_{y}^{t}=\mathscr{O}_{\mathscr{Z},(t, y)} \otimes_{\mathcal{O}_{\mathscr{Z},(t, y)}} \mathrm{C}
$$

for every $y$ in $Y_{t}$. It follows that

$$
\operatorname{dim}_{C} \mathscr{A}_{y}^{t}=\operatorname{dim}_{\mathscr{O}_{T, t}} \mathscr{O}_{\mathscr{Z},(t, y)}
$$

and that

$$
\sum_{y \in Y_{t}} n_{y}(t)=k
$$

for every $t$ in $T$. Hence $X_{t} \in \operatorname{Sym}^{k}(B)$ for all $t$ in $T$ and the proof of the theorem consists of showing that the mapping

$$
T \rightarrow \operatorname{Sym}^{k}(B), \quad t \mapsto X_{t}
$$

is holomorphic.

As before let $q: \mathscr{Z} \rightarrow B$ denote the canonical projection and write $q=$ $\left(q_{1}, \ldots q_{p}\right)$. Each $q_{j}$ is a global holomorphic function on $\mathscr{Z}$ so multiplication by $q_{j}$ defines a $\mathscr{O}_{T}$-linear endomorphism

$$
\pi_{*} \mathscr{O}_{\mathscr{Z}} \rightarrow \pi_{*} \mathscr{O}_{\mathscr{Z}}
$$

and for each $t$ in $T$ it induces a C-linear endomorphism

$$
L_{j}: \mathscr{A}^{t} \rightarrow \mathscr{A}^{t}
$$

In terms of the decomposition

$$
\mathscr{A}^{t}=\bigoplus_{y \in Y_{t}} \mathscr{A}_{y}^{t}
$$

we obviously have for every $j$ that

$$
L_{j}\left(\sum_{y \in Y_{t}} v_{y}\right)=\sum_{y \in Y_{t}} q_{j}(t, y) v_{y}
$$

so for each $y$ in $Y_{t}$ every non-zero element of $\mathscr{A}_{y}^{t}$ is an eigenvector for $L_{j}$ corresponding to the eigenvalue $q_{j}(t, y)$. 
Now let $t$ be a fixed point in $T$. Since the $\mathscr{O}_{T, t}$-algebra $\left(\pi_{*} \mathscr{O}_{\mathscr{L}}\right)_{t}$ is a free $\mathscr{O}_{T, t^{-}}$ module the polynomial algebra $\left(\pi_{*} \mathscr{O}_{\mathscr{L}}\right)_{t}\left[X_{1}, \ldots, X_{p}\right]$ is a free $\mathscr{O}_{T, t}\left[X_{1}, \ldots\right.$, $\left.X_{p}\right]$-module. Put

$$
P:=q_{1} X_{1}+\cdots+q_{p} X_{p}
$$

and consider it as an element in $\mathscr{O}_{\mathscr{Z}}(\mathscr{Z})\left[X_{1}, \ldots, X_{p}\right]$. Multiplication by $P$ defines a $\mathscr{O}_{T, t}\left[X_{1}, \ldots, X_{p}\right]$-linear endomorphism

$$
\mathscr{L}:\left(\pi_{*} \mathscr{O}_{\mathscr{L}}\right)_{t}\left[X_{1}, \ldots, X_{p}\right] \rightarrow\left(\pi_{*} \mathscr{O}_{\mathscr{L}}\right)_{t}\left[X_{1}, \ldots, X_{p}\right]
$$

whose characteristic polynomial is of the form

$$
\lambda^{k}+\alpha_{1} \lambda^{k-1}+\cdots+\alpha_{k-1} \lambda+\alpha_{k}
$$

where $\alpha_{j}$ is a homogeneous polynomial of degree $j$ in $\mathscr{O}_{T, t}\left[X_{1}, \ldots, X_{p}\right]$.

For each $t$ in $T$ the polynomial

$$
\lambda^{k}+\alpha_{1}(t) \lambda^{k-1}+\cdots+\alpha_{k-1}(t) \lambda+\alpha_{k}(t)
$$

is the characteristic polynomial of the $\mathrm{C}\left[X_{1}, \ldots, X_{p}\right]$-linear endomorphism

$$
\mathscr{L}(t): \mathscr{A}^{t}\left[X_{1}, \ldots, X_{p}\right] \rightarrow \mathscr{A}^{t}\left[X_{1}, \ldots, X_{p}\right]
$$

defined by multiplication by the polynomial

$$
P(t):=q_{1}(t, \cdot) X_{1}+\cdots+q_{p}(t, \cdot) X_{p} .
$$

From our considerations above concerning the endomorphisms $L_{1}, \ldots, L_{p}$ we see that the characteristic polynomial $(*)$ has $k$ different roots in $\mathrm{C}\left[X_{1}, \ldots, X_{p}\right]$, namely

$$
q_{1}(t, y) X_{1}+\cdots+q_{p}(t, y) X_{p},
$$

where $y$ ranges over the $k$ points in $Y_{t}$ (counted with multiplicities). Hence $\alpha_{1}(t), \ldots, \alpha_{k}(t)$ are the symmetric polynomials of these roots and this proves that the holomorphic map

$$
T \rightarrow \bigoplus_{j=1}^{k} S_{j}\left(\mathbf{C}^{p}\right), \quad t \mapsto\left(\alpha_{1}(t), \ldots, \alpha_{k}(t)\right)
$$

is the composition of the map

$$
T \rightarrow \operatorname{Sym}^{k}(B), \quad t \mapsto X_{t}
$$

and the holomorphic embedding $\operatorname{Sym}^{k}(B) \rightarrow \bigoplus_{j=1}^{k} S_{j}\left(C^{p}\right)$ described in section 1. It then follows that the former map is holomorphic and the theorem is proved. 


\section{The general case}

For the proof of our Main Theorem (stated in the introduction) we need the following results.

Lemma 3.1. Let $S$ be a reduced complex space, let $U$ be an open polydisk in $\mathrm{C}^{n}$, let $\Sigma$ be a closed subset of $S \times U$ such that $\Sigma \cap(\{s\} \times U)$ is contained in a nowhere dense analytic subset of $\{s\} \times U$ for all $s$ in $S$ and let

$$
g: S \times U \backslash \Sigma \rightarrow \mathrm{C}
$$

be a holomorphic function. If the restriction of $g$ to $\{s\} \times U \backslash \Sigma$ extends holomorphically to $\{s\} \times U$ for all $s$ in $S$, then $g$ extends holomorphically to $S \times U$.

Proof. The proof is based on Cauchy's formula. See [1] for details.

Lemma 3.2. Let $A$ and $B$ be local Noetherian rings and $\rho: A \rightarrow B$ be a local homomorphism such that $B$ is a flat A-module via $\rho$. Let a be a proper ideal of $A$ and let $\mathfrak{b}$ denote the ideal generated by $\rho(\mathfrak{a})$ in $B$. Then for every finitely generated $B$-module $M$ the following conditions are equivalent:

(i) $M$ is a flat $B$-module.

(ii) $M$ is a flat $A$-module and $M / \mathfrak{b} M$ is flat $B / \mathfrak{b}$-module.

Proof. See [2].

Proof of the Main Theorem. Since base change respects flatness we may assume without loss of generality that the space $S$ is reduced. Let $s_{0}$ be a point in $S$ and let $E=(U, B, j)$ be a scale of $M$ adapted to $Z_{s_{0}}$, i.e.

$$
j^{-1}(\bar{U} \times \partial B) \cap Z_{s_{0}}=\emptyset .
$$

Since $j^{-1}(\bar{U} \times \partial B)$ is compact there exists an open neighbourhood $S_{0}$ of $s_{0}$ in $S$ such that

$$
j^{-1}(\bar{U} \times \partial B) \cap Z_{s}=\emptyset
$$

for all $s$ in $S_{0}$. Then

$$
Y:=\left(\operatorname{id}_{S_{0}} \times j\right)\left(\left(S_{0} \times j^{-1}(U \times B)\right) \cap \mathscr{Z}\right)
$$

is a complex $n$-dimensional subspace of $S_{0} \times U \times B$ that satisfies the following conditions:

- The canonical projection $f: Y \rightarrow S_{0}$ is flat.

- The canonical projection $\pi: Y \rightarrow S_{0} \times U$ is finite. 
For each $s$ in $S_{0}$ the fiber of $f$ over $s$ will be denoted by $Y_{s}$ and $\{s\} \times U$ will often be identified with $U$ to simplify the presentation.

Denote by $\Sigma$ the set of all points in $S_{0} \times U$ where the coherent $\mathcal{O}_{S_{0} \times U^{-}}$ module $\pi_{*} \mathscr{O}_{Y}$ is not free. Then $\Sigma$ is a thin analytic subset of $S_{0} \times U$. See [4] for instance.

Let us first show that $\Sigma \cap(\{s\} \times U)$ is a thin analytic subset of $\{s\} \times U$ for all $s \in S_{0}$. For $s \in S_{0}$ and $u \in U$, put $M:=\left(\pi_{*} \mathscr{O}_{Y}\right)_{(s, u)}, B:=\mathscr{O}_{S_{0} \times U,(s, u)}$, $A:=\mathcal{O}_{S_{0}, s}$ and let $a$ be the maximal ideal of $A$. Then by lemma 3.2 we get:

$\left(\pi_{*} \mathcal{O}_{Y_{s}}\right)_{u}$ is a free $\mathcal{O}_{U, u}$-module if and only if $\left(\pi_{*} \mathcal{O}_{Y}\right)_{(s, u)}$ is a free $\mathcal{O}_{S \times U,(s, u)^{-}}$ module.

It follows that $\Sigma \cap(\{s\} \times U)$ is a thin analytic subset of $\{s\} \times U$ for all $s \in S_{0}$.

For $s \in S_{0}$, denote by $Y_{s}^{\prime}$ the union of those irreducible components of $Y_{s}$ whose dimension is strictly less than $n$ and denote by $\bar{Y}_{s}$ the analytic closure of $Y_{s} \backslash Y_{s}^{\prime}$ in $Y_{s}$. Let us show that $\pi\left(Y_{s} \backslash \bar{Y}_{s}\right) \subset \Sigma$.

If $y \in Y_{s} \backslash \bar{Y}_{s}$ then $\mathcal{O}_{Y_{s}, y}$ is not a free module over $\mathscr{O}_{U, \pi(y)}$ since $\operatorname{dim}_{y} Y_{s}<$ $n=\operatorname{dim} U$. It follows that $\left(\pi_{*} \mathcal{O}_{Y_{s}}\right)_{\pi(y)}$ is not a free $\mathscr{O}_{U, \pi(y)}$-module so by lemma 3.2 the $\mathscr{O}_{S_{0} \times U, \pi(y)}$-module $\left(\pi_{*} \mathscr{O}_{Y}\right)_{\pi(y)}$ is not free. Hence $\pi(y) \in \Sigma$. This shows that $\pi\left(Y_{s} \backslash \bar{Y}_{s}\right) \subset \Sigma$ for all $s \in S_{0}$.

Put $T:=S_{0} \times U \backslash \Sigma$. Then

$$
\pi^{-1}(T) \subset T \times B
$$

is a flat and proper family of 0-dimensional subspaces of $B$ and by theorem 2.1 the corresponding family of fundamental cycles is an analytic family of 0 cycles in $B$. Let

$$
g: T \rightarrow \operatorname{Sym}^{k}(B)
$$

be the holomorphic map defined by that family, where $k$ is the rank of the $\mathcal{O}_{S_{0} \times U}$-module $\pi_{*} \mathscr{O}_{Y}$. For every $s$ in $S_{0}$ the holomorphic map

$$
g(s, \cdot):\{s\} \times U \backslash \Sigma \rightarrow \operatorname{Sym}^{k}(B)
$$

extends to the holomorphic map induced by the fundamental cycle $\left[Z_{s}\right]$ in the scale $E$. From this and lemma 3.1 we deduce that the map $g$ extends to a holomorphic map

$$
\hat{g}: S_{0} \times U \rightarrow \operatorname{Sym}^{k}(B)
$$

such that $\hat{g}(s, \cdot): U \rightarrow \operatorname{Sym}^{k}(B)$ is the holomorphic map induced by $\left[Z_{s}\right]$ in the scale $E$. Hence $\left(\left[Z_{s}\right]\right)_{s \in S}$ is an analytic family of $n$-cycles and the proof is completed. 
COROLlary 3.3. Let $M$ be a complex space. Then the mapping

$$
\mathscr{D}(M)_{\text {red }} \rightarrow \mathscr{C}(M), \quad Z \rightarrow[Z]
$$

is holomorphic.

Proof. It is easy to see that the complex subspaces of $M$ belonging to the same connected component of $\mathscr{D}(M)$ are all of the same dimension. Thus we only have to show that for any flat and proper family of subspaces of a certain dimension in $M$ the corresponding analytic family of fundamental cycles is also proper.

Let $(S, \mathscr{Z})$ be a flat and proper family of $n$ dimensional subspaces of $M$. To show that the corresponding analytic family of the fundamental $n$-cycles is proper we may assume, without loss of generality, that the space $S$ is reduced and (globally) irreducible. Put $d:=\operatorname{dim} S$ and let $\pi: \mathscr{Z} \rightarrow S$ be the canonical projection. Since $\pi$ is flat the equality

$$
\operatorname{dim}_{z} \mathscr{Z}=\operatorname{dim}_{z} \mathscr{Z}_{\pi(z)}+d
$$

holds for every $z \in \mathscr{Z}$. It follows easily that the graph of the corresponding analytic family is the union of all irreducible components of dimension $n+d$ of $\mathscr{Z}$. The restriction of $\pi$ to this subspace of $\mathscr{Z}$ being proper the proof is completed in virtue of the remark following definition 1.4 .

\section{REFERENCES}

1. Barlet, D., Familles de cycles analytiques paramétrées par un espace analytique réduit, Lecture Notes in Math. 482 (1975), 1-158.

2. Bourbaki, N., Commutative Algebra, Hermann (1972).

3. Douady, A., Le problème des modules pour les sous-espaces analytiques compacts d'un espace analytique donné, Ann. Inst. Fourier (Grenoble) 16, 1 (1966), 1-95.

4. Fischer, G., Complex Analytic Geometry, Lecture Notes in Math. 538 (1976).

5. Kaup, B., Kaup, L., Holomorphic Functions of Several Variables, de Gruyter Berlin New York (1983).

6. Mumford, D., Fogarty, J., Geometric Invariant Theory, Ergeb. Math. Grenzgeb. 34 (1982).

7. Whitney, H., Complex Analytic Varieties, Addison-Wesley (1972).

SCIENCE INSTITUTE

UNIVERSITY OF ICELAND

REYKJAVÍK

ICLEAND

E-mail: jim@raunvis.hi.is 